\title{
AIOM RECOMMENDATIONS ON THE USE OF CYTOREDUCTIVE SURGERY AND HIPEC IN PRIMARY AND SECONDARY PERITONEAL TUMORS
}

\author{
A. Damato ${ }^{1,2^{*}}$, F. Petrelli ${ }^{3}$, M. Deraco ${ }^{4}$, M. Di Bartolomeo ${ }^{5}$, M. De Simone ${ }^{6}$, \\ G. Zannoni ${ }^{7}$, L. Ansaloni ${ }^{8}$, A. Laghi ${ }^{9}$, A. Sommariva ${ }^{10}$, A. Fagotti ${ }^{11}$, \\ D. Bellini ${ }^{12}$, C. Pinto ${ }^{1}$ \\ ${ }^{1}$ Medical Oncology Unit, Azienda USL-IRCCS di Reggio Emilia, Reggio Emilia, Italy \\ 2 Department of Medical Biotechnologies, University of Siena, Siena, Italy \\ ${ }^{3}$ Oncology Unit, ASST Bergamo Ovest, Treviglio, Italy \\ ${ }^{4}$ Peritoneal Malignancy Program, Department of Surgery, Fondazione IRCCS Istituto Nazionale dei Tumori, Milan, Italy \\ ${ }^{5}$ Department of Medical Oncology, Fondazione IRCCS Istituto Nazionale dei Tumori, Milan, Italy \\ ${ }^{6}$ Department of Surgical Oncology, Candiolo Institute for Cancer Research and Treatment, Turin, Italy \\ ${ }^{7}$ Human Pathology, Pathology Department, Catholic University of the Sacred Heart, Rome, Italy \\ ${ }^{8}$ General Surgery Unit, University of Pavia, Pavia, Italy \\ ${ }^{9}$ Radiology Unit-Sant'Andrea University Hospital, Department of Surgical and Medical Sciences and Translational \\ Medicine, Sapienza University of Rome, Rome, Italy \\ ${ }^{10}$ Advanced Surgical Oncology Unit, Surgical Oncology of the Esophagus and Digestive Tract, Veneto Institute of \\ Oncology IOV-IRCCS Padua, Italy \\ ${ }^{11}$ Department of Woman, Child and Public Health, Fondazione Policlinico Universitario A. Gemelli IRCCS, Rome, Italy \\ 12 Department of Radiological Sciences, Oncology and Pathology, Sapienza University of Rome - I.C.O.T. Hospital, \\ Latina, Italy
}

\section{CORRESPONDING AUTHOR:}

Angela Damato

Medical Oncology Unit

Azienda USL-IRCCS di Reggio Emilia

viale Risorgimento 80

42123 Reggio Emilia, Italy

E-mail: angela.damato@ausl.re.it

ORCID: 0000-0001-8286-1274

Doi: $10.48286 / a r o .2021 .21$

History

Received: Jul 21, 2021

Accepted: Aug 27, 2021

Published: Sept 1, 2021

\section{ABSTRACT}

Peritoneal surface malignancies represent rare and hard-to-treat entities that include primarily abdominal that involve disseminating cancer cells into abdominal peritoneum, with or without associated extraperitoneal disease. Diagnosis and management of these

aggressive cancers need a dedicated multidisciplinary team and a high-volume center for locoregional treatment where technically and clinically feasible.

This article summarizes the most updated evidence-based guidelines that the Italian Medical 
Oncology Association (AIOM) has implemented with a multidisciplinary panel of experts, including dedicated expert clinicians such as pathologists, surgeons, medical oncologists, and the support of methodologists, to guide clinicians involved in the primary management of patients with peritoneal neoplasms in their daily clinical practice. Based on the type of studies addressing the questions and their methods, AIOM guideline methodologists used the GRADE method (Grading of Recommendations Assessment, Development, and Evaluation: GRADE) to classify the quality of each kind of evidence.

In selected cases, the main curative treatment consists of a cytoreduction surgery (CRS) that implies

\section{KEY WORDS}

Peritoneal tumors; cytoreductive surgery; HIPEC; guidelines. the complete removal of the macroscopically appreciable disease or any minimum residual millimeter. It is then associated with intraperitoneal chemo-hyperthermia (HIPEC), carried out at the end of the surgical demolition time, a particular type of chemotherapy that exploits the combined effect of heat and high concentrations of drugs, with a localized action in the area affected by the neoplasm.

We here provide recommendations for 6 main clinical scenarios: primary treatment of primary serous peritoneal papillary carcinoma, pseudomixoma peritonei, colorectal and gastric cancer, ovarian carcinoma, and peritoneal mesothelioma.

\section{IMPACT STATEMENT}

This paper represents a synthesis of 2021 clinical practice guidelines about presentation, diagnosis and management of primary and secondary peritoneal surface malignancies provided by an expert panel on behalf of AIOM.

cal practice guidelines for primary and secondary peritoneal tumors (1), which were subsequently updated in 2020. This article summarizes the most updated evidence-based guidelines that the AIOM has implemented with a multidisciplinary panel of experts, including dedicated expert clinicians such as pathologists, surgeons, medical oncologists, and the support of methodologists, to guide clinicians involved in the primary management of patients with peritoneal neoplasms in their daily clinical practice. Based on the type of studies addressing the questions and their methods, AIOM guideline methodologists used the GRADE method (Grading of Recommendations Assessment, Development, and Evaluation: GRADE) to classify the quality of each kind of evidence. In particular, the GRADE method assesses methodological bias within the studies, uniformity between different studies results; consistency of results across different studies; repeatability of results on a broader patient sample set; the effectiveness of treatments. Treatment comparisons result in one out of four GRADE scores, reflecting the quality of the 


\begin{tabular}{|c|c|c|}
\hline $\begin{array}{l}\text { STRONGNESS } \\
\text { OF CLINICAL } \\
\text { RECOMMENDATION }\end{array}$ & TERMS & MEANING \\
\hline STRONG POSITIVE & $\begin{array}{l}\text { Strong Positive "In patients } \\
\text { with (selection criteria) the xxx } \\
\text { intervention should be considered as } \\
\text { a first intention therapeutic option". }\end{array}$ & $\begin{array}{l}\text { The intervention in question should be } \\
\text { considered as the first therapeutic option } \\
\text { (evidence that the benefits outweigh the harm). }\end{array}$ \\
\hline WEAK POSITIVE & $\begin{array}{l}\text { "In patients with (selection } \\
\text { criteria), the xxx intervention can } \\
\text { be considered as a first intention } \\
\text { therapeutic option, as an alternative } \\
\text { to yyy". }\end{array}$ & $\begin{array}{l}\text { The intervention in question can be considered } \\
\text { as a first intention option, aware of the existence } \\
\text { of alternatives equally feasible (uncertainty } \\
\text { regarding the prevalence of benefits over } \\
\text { damages). }\end{array}$ \\
\hline WEAK NEGATIVE & $\begin{array}{l}\text { "In patients with (selection criteria), } \\
\text { the xxx intervention should not } \\
\text { be considered as a first intention } \\
\text { treatment option, as an alternative to } \\
\text { yyy". }\end{array}$ & $\begin{array}{l}\text { The intervention in question should not be } \\
\text { considered as a first intention option; it could, } \\
\text { however, be suitable for use in highly selected } \\
\text { cases and after complete sharing with the patient } \\
\text { (uncertainty regarding the prevalence of harm } \\
\text { over benefits). }\end{array}$ \\
\hline STRONG NEGATIVE & $\begin{array}{l}\text { "In patients with (selection criteria), } \\
\text { the xxx intervention must not be } \\
\text { considered as a first intention } \\
\text { therapeutic option". }\end{array}$ & $\begin{array}{l}\text { The intervention in question must in no case be } \\
\text { taken into consideration (evidence that the harm } \\
\text { prevails over the benefits). }\end{array}$ \\
\hline
\end{tabular}

Table I. Strongness of clinical recommendation graded in four levels based on clinical relevance.

evidence: high-quality, moderate-quality, low-quality, or very low-quality evidence. The strength of the recommendation is graded, based on clinical importance, on four levels (table I).

The patient's clinical history greatly conditions the diagnostic classification of peritoneal neoplasms. Specifically, the cases in which a peritoneal neoplastic pathology is diagnosed during the follow-up of a known primary neoplasm should be distinguished from those in which the patient's medical history is mute. In the first case, the diagnostic process aims to verify the correlation between the metastatic event and previous cancer. In the second case, however, the diagnostic workout must be planned according to the invasiveness of the procedures and resources availability.

The diagnostic process must first consider the more frequent pathologies according to the sex and age of the patient. The other diagnostic elements that emerge from clinical evaluations, blood chemistry, and instrumental tests (tumor markers, computed tomography, PET-CT, MRI, endoscopies) must be integrated with the epidemiological data. The indication for the surgical, diagnostic procedure (laparoscopy or exploratory laparotomy) must be placed with extreme accuracy and if the other diagnostic procedures could not lead to the diagnosis of certainty (figure 1). These invasive procedures allow both the biopsy of neoplastic material and an accurate estimate of the extent of peritoneal disease using the Peritoneal Cancer Index $(\mathrm{PCl}) . \mathrm{PCl}$ is a value determined by the size of the peritoneal implants and the distribution of nodules on the peritoneal surface. For the evaluation of the final score, the size of the peritoneal nodules is first assessed; The sum of the lesion size score and the distribution of tumor in the abdominopelvic regions gives us the patient's $\mathrm{PCl}$. Implants are scored as lesion size 0 through 3 (LS0 to LS-3). LS-0: no implants are seen throughout the region; LS-1: implants visible up to $0.5 \mathrm{~cm}$ in greatest diameter; LS-2: nodules greater than 0.5 $\mathrm{cm}$ and up to $5 \mathrm{~cm}$; LS-3: implants $5 \mathrm{~cm}$ or greater in diameter (2). However, it is not recommended to proceed with aggressive surgical attempts and debulking before a complete and correct diagnostic classification. Adverse events resulting from improper abdominal-pelvic surgical manipulation are worrisome. They include disseminating neoplastic cells in the surgical explored areas that remain trapped in fibrosis and fibrin and are may become poorly sensitive to systemic and local-regional chemotherapy treatments, inevitably leading to cell proliferation and neoplastic growth (3-7).

Cytoreduction surgery (CRS) implies the concept of surgical radicality with the complete removal 


\section{Diagnostic framework of peritoneal carcinomatosis}

Clinical-instrumental suspicion of peritoneal carcinosis with or without ascites with negative history of oncological disease

THORAX AND ABDOMINAL CT SCAN

\section{Isolated carcinosis in the absence of other metastatic sites}

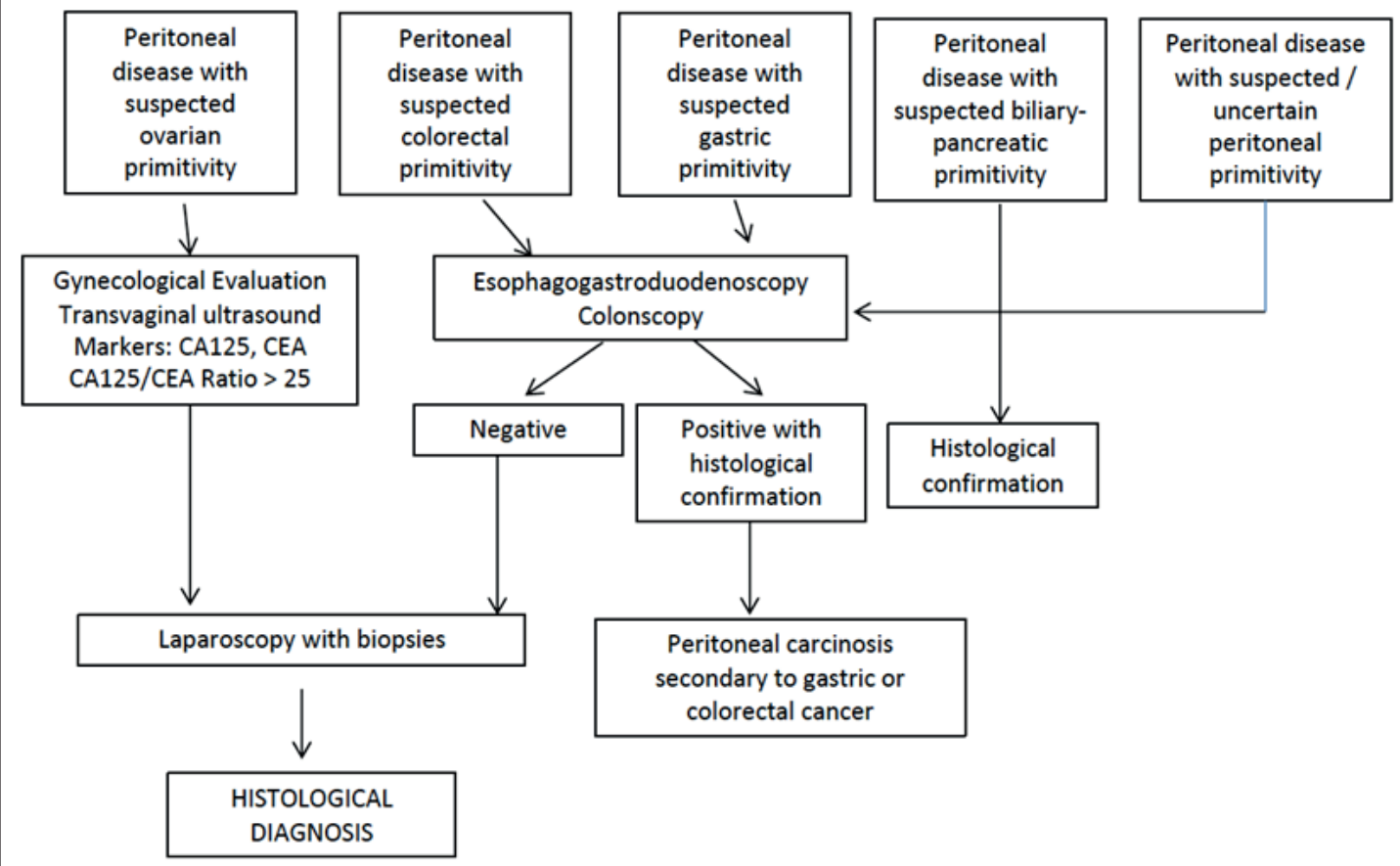

Figure 1. Diagnostic workup of peritoneal carcinomatosis.

of the macroscopically appreciable disease or any minimum residual millimeter. This concept differs from debulking, which implies palliative removal of part of the neoplastic disease with gross neoplastic residue. CRS for peritoneal tumors is a concept developed by Paul H. Sugarbaker (8). The completeness of Cytoreduction (CCR) is coded at the end of the surgical phase according to the criteria validated by the Consensus Conference of 2006 ( 9 , 10). It represents the most important prognostic factor of peritoneal neoplasms and is expressed by cc-score: CC-0 (absence of visible residue), CC-1 (residue lower than $2.5 \mathrm{~mm}$ ), CC-2 (residue greater than $2.5 \mathrm{~mm}$ and less than $5 \mathrm{~cm}$ ), CC-3 (residue greater than $5 \mathrm{~cm}$ and with confluent nodules).

Intraperitoneal chemo-hyperthermia (HIPEC), carried out at the end of the surgical demolition time, represents a locoregional therapeutic aid, supplementary to the surgical intervention; its action is manifested in the moment of maximum cytoreduction of the neoplasm, in the absence of adhesions, and above all before the cells released in the abdomen are implanted on the bloody surfaces or are protected by the physiological deposition of fibrin and stimulated to proliferation by the chemical mediators of inflammation. It is a particular type of chemotherapy that exploits the combined effect of heat and high concentrations of drugs, with a localized action in the area affected by the neoplasm. It consists in the perfusion of the abdominal cavity with a variable quantity (3-6 liters) of liquid (Perfusate) in which high doses of chemotherapy are administered in conditions of hyperthermia. HIPEC is performed immediately after finishing the CRS by placing a system of cannulas through the abdominal wall connected to an extracorporeal circulation circuit. Therefore, the perfusate is circulated with chemotherapeutic agents in conditions of hyperthermia with a closed abdomen or an open abdomen. Duration of perfusion, type of chemotherapy and temperatures are a function of the histological type to be treated $(11,12)$. 


\section{RECOMMENDATIONS FOR CRS AND HIPEC}

In patients with Primary Serous Peritoneal Papillary Carcinoma (SPPC), the neoadjuvant chemotherapy followed by HIPEC associated with CRS is indicated compared to chemotherapy treatment followed by interval debulking surgery? (table II)

SPPC is histologically similar to epithelial ovarian cancer (EOC) but clinically differs by a predominantly peritoneal widespread, with a little ovarian involvement. The epidemiological, clinical and molecular differences between SPPC and EOC have been highlighted and described in a review (13). The exact incidence of SPPC is not clear, and actually, about $10-20 \%$ of EOC labeled as serous papillary ovarian carcinoma are SPPC.

Due to the similarities with EOC, SPPC has often been treated by surgery and systemic chemotherapy (sCT) containing platinum and taxanes. Therefore, a lot of data arises from small retrospective cohorts or case-control, comparing patients with SPPC and EOC. The median overall survival (mOS) of patients with SPPC is 21-42 months, shorter than EOC patients, with a progression-free survival (PFS) of 11-17 months (14-16).

The experience gained in other peritoneal neoplasms through CRS and HIPEC has motivated various groups to extend the indications also on SPPC as a primary peritoneal neoplasm. The rationale for this approach is based on the multifocality, polyclonality, and the high frequency of widespread peritoneal metastases of SPPC. The analysis of 36 patients with SPPC treated with CRS and HIPEC was conducted in France and Italy (17). In addition, 35 patients received platinum-based systemic adjuvant treatment. Morbidity and mortality were $20.6 \%$ and $5.6 \%$, respectively. Five-year OS was $57.4 \%$ and DFS was 24\% (median of 16.7 months). A single-center analysis of 29 patients with SPPC homogeneously treated with neoadjuvant chemotherapy with 6 cycles of Carboplatin and Paclitaxel followed by CRS and HIPEC, after a median follow-up of 12 months, showed a 5-years OS of $64.9 \%$ (median not reached) $(18,19)$. Overall, grade III-IV surgical complication was seen in 4/22 (18\%) patients; no post-operative mortality was observed. Median PFS was 32.9 months, and 5-year PFS was $33.2 \%$. CRS was performed with total parietal peritonectomy and with HIPEC using a chemotherapy combination based on Cisplatin plus Doxorubicin at 43 Celsius degree of temperature for $90 \mathrm{~min}$ utes.

Based on these results, the absence of randomized studies comparing the integrated treatment to standard chemotherapy and surgical debulking limits the significance of the results. However, the benefit assessed as overall and progression-free survival of SCT plus CRS and HIPEC treatment and relatively limited adverse events compared to SCT and surgical debulking, get the judgment in favor of the benefit over the damage, but it must be discussed with the patient regarding the extension of the surgical treatment. Therefore, the panel provided a positive recommendation in favor of SCT plus CRS and HIPEC.

In patients with resectable pseudomyxoma peritonei, is HIPEC associated with cytoreduction indicated rather than surgical debulking and systemic chemotherapy?

The main supporting literature included the McBride, et al. study (20), which is a review and meta-analysis of 15 observational studies concerning the treatment of pseudomyxoma peritonei by CRS associated with HIPEC in various forms (EPIC, HIPEC, HIPEC + EPIC). Median survival at 3, 5, and 10 years was $77.85 \%, 79.5 \%$, and $55.9 \%$, respectively. The median complication rate (calculated across 14 studies) was $40 \%$. Although the complication rate is not negligible, the panel believes that the benefit given by CRS associated with HIPEC is still higher than that provided by repeated debulking surgeries.

Based on these assessments and experience in the field, the AIOM panel unanimously judged the balance between risks and benefits deriving from the execution of CRS and HIPEC in resectable pseudomyxoma peritonei to be favorable and provided a weak positive recommendation in favor of intervention.

If operability and resectability criteria are met in patients with diffuse malignant epithelioid peritoneal mesothelioma, are cytoreductive and HIPEC procedures indicated compared to systemic chemotherapy?

In patients with peritoneal mesothelioma, the efficacy of the combined treatment with CRS and HIPEC is reported in numerous papers reported in the literature (21-24). In summary, the results offered by palliative chemotherapy alone with convention- 


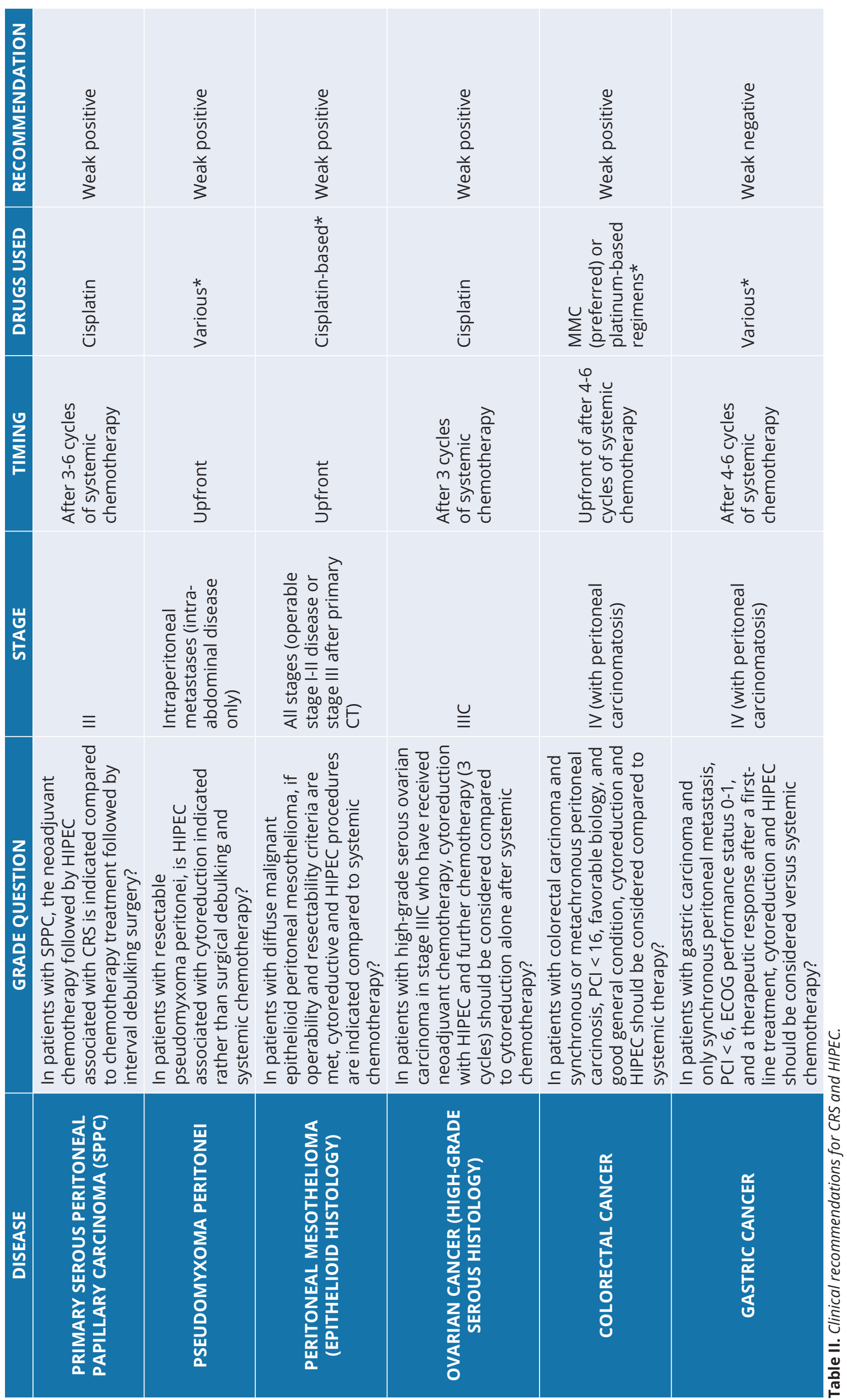


al agents (pemetrexed and cisplatin) are extremely disappointing (median OS < 8-10 months). In a systematic review including 20 observational studies, Helm et al. analyzed a total of 1,047 patients with peritoneal mesothelioma with median $\mathrm{PCl}$ of 19 (25). An optimal intervention of CRS (complete cytoreduction-0/1) + HIPEC was performed in $67 \%$ of patients. Overall survival at 1,3 , and 5 years was respectively 84,59 , and $42 \%$, being significantly higher than historical data with traditional treatment. Deraco, et al. reported an overall and progression-free 5-year survival of $57 \%$ and $31 \%$, respectively, in a single-center series (26). The postoperative grade 3 morbidity was $15 \%$ in the absence of mortality correlated with the surgery, while the toxicity resulting from the chemo-hyperthermic treatment was $12 \%$. Surgical radicality, performance status, and mitotic counts were statistically correlated with the results. In a further monocentric experience reported by Robella et al. (27), the OS at 1 and 5 years was $63 \%$ and $44 \%$, respectively, with an overall morbidity rate of $35.7 \%$ associated with a perioperative mortality of $7.1 \%$. Regarding the quality of life after HIPEC CRS treatment, the potential high morbidity correlated with the complexity of the surgical procedure must be taken into account. In the experience of Piso et al. (28), even if the published data show a compromise in the postoperative quality of life at three months after surgery, there is subsequently an improvement over 6-12 months to levels higher than baseline. The evidence of the results relating to survival and quality of life despite the frequency of adverse events allowed the panel to unanimously judge a positive balance between benefits and risks deriving from the execution of CRS and HIPEC and recommended in favor of intervention.

In patients with high-grade serous carcinoma of the ovary in stage IIIC who have received neoadjuvant chemotherapy, should CRS + HIPEC and further chemotherapy ( 3 cycles) be considered an alternative to CRS alone after systemic chemotherapy?

The Netherlands Cancer Institute conducted a large, randomized, open-label phase III study by Van Driel et al. Their results were published in the New England Journal of Medicine in January 2018 (29). The trial was conducted on 245 patients with stage III serous ovarian cancer and included, after three courses of neoadjuvant chemotherapy with carboplatin and paclitaxel, a 1: 1 randomization with CRS only ( $n=123$ patients) versus CRS plus
HIPEC with Cisplatin $100 \mathrm{mg} / \mathrm{m} 2$ alone ( $\mathrm{n}=122$ patients). Subsequently, adjuvant treatment with systemic chemotherapy was delivered in both groups for three cycles with carboplatin and paclitaxel. The primary endpoint was relapse-free survival (RFS). The median RFS was 10.7 months in the surgery-only group versus 14.2 months in the CRS plus HIPEC group. At a median follow-up of 4.7 years, mortality was higher in the CRS group (62\% of patients) than in the CRS and HIPEC group ( $50 \%$ of patients) (hazard ratio, $0.67 ; 95 \% \mathrm{Cl}, 0.48$ to $0.94 ; p=0.02$ ). The median survival, secondary endpoint, was higher in the experimental CRS plus HIPEC group (45.7 months) than in the CRS alone group (39.9 months). The percentage of grade 3-4 adverse events reported in the two groups was almost overlapping, respectively $25 \%$ in the CRS group and $27 \%$ in the CRS plus HIPEC group ( $p=$ 0.76 ), supporting the feasibility and tolerability of the integrated procedure.

In consideration of the available data, AIOM judged favorable the balance between risks and benefits and provided a positive recommendation for the execution of CRS associated with HIPEC and subsequent systemic chemotherapy (3 cycles) after disease control with neoadjuvant chemotherapy in patients with high-grade, stage III, serous ovarian carcinoma. However, in patients eligible for this approach, such treatment should be carried out at high-volume centers where high expertise is expected.

Should CRS and HIPEC be considered the only alternative treatment to systemic therapy in patients with colorectal carcinoma and synchronous or metachronous peritoneal carcinosis, $\mathrm{PCl}<16$, favorable biology, and good general condition?

In a small open-label randomized Swedish study by Cashin et al. published in the European Journal of Cancer in 2016 and closed prematurely for poor accrual, 48 patients were randomized to receive CRS plus HIPEC with 5-FU versus oxaliplatin-based chemotherapy alone (30). Median survival was 25 vs. 18 months in favor of the experimental arm (RR $0.79,95 \% \mathrm{Cl} 0.64-0.97$ ) with a $21 \%$ reduction in the absolute risk of death. However, the progression-free survival (PFS) was lower in magnitude (RR $0.83,95 \% \mathrm{Cl} 0.7-1)$. Against these efficacy data, no fatal events occurred at 30 days (strong evidence). 12 serious adverse events were reported in 10 patients in the experimental arm, compared with 14 grade $3-4$ events reported in 12 patients in the 
chemotherapy arm, with overall low evidence of consistency of the toxicity.

For this reason, the panel unanimously judged favorable the balance between risks and benefits deriving from the execution of CRS and HIPEC in peritoneal carcinosis from synchronous or metachronous colorectal carcinoma $\mathrm{PCl}<16$, favorable biology and good general conditions and provide a weak positive recommendation in favor of intervention.

Should CRS plus HIPEC versus systemic chemotherapy be used for patients with gastric carcinoma and only synchronous peritoneal metastasis, $\mathrm{PCl}<6$, ECOG performance status 0-1, and a therapeutic response after a first-line treatment?

Peritoneal carcinomatosis of gastric origin is recognized as an independent poor prognostic factor associated with poor prognosis. Systemic chemotherapy options do not differ from those of metastatic disease, although carcinosis is a factor associated with poor response to systemic treatment, mainly due to poor bioavailability of drugs on the peritoneal surface. According to the current state of evidence and literature data, although it represents a field of research in high-volume centers, the locoregional treatment with CRS and HIPEC in gastric adenocarcinoma with peritoneal carcinomatosis does not seem to represent a recommended treatment at least in the Western population. Although one randomized study (31) demonstrates a non-significant trend in favor of higher five years OS, the confidence intervals are broad and similar results are also reported for PFS. The panel, therefore, believes that the expected desirable effects (prolongation of OS and RFS / PFS) resulting from the integrated treatment of CRS and HIPEC in the PS 0-1 patient with peritoneal carcinosis alone from primary gastric cancer and $\mathrm{PCl}<6$, are negligible and so provide strong negative recommendation against it.

\section{ETHICS}

\section{Fundings}

There were no institutional or private fundings for this article.

\section{Conflict of interests}

The authors have declared no conflict of interests.

\section{Availability of data and material \\ N/A}

\section{Authors' contribution}

All the authors contributed equally to conception, data collection, analysis of this paper. AD and FP wrote this article.

\section{Ethical approval \\ N/A}




\section{REFERENCES}

1. Available from: https://www.aiom.it/wp-content/uploads/2018/11/2018_LG_AIOM_Peritoneali.pdf.

2. Gilly FE, Cotte E, Brigand C, et al. Quantitative prognostic indices in peritoneal carcinomatosis. Eur Surg J Oncol 2006;(6):597-601.

3. Kusamura S, Kepenekian V, Villeneuve L, et al. PSOGI Peritoneal mesothelioma: PSOGI/EURACAN clinical practice guidelines for diagnosis, treatment and follow-up. Eur J Surg Oncol 2020;S0748-7983(20)30113-X.

4. Govaerts K, Lurvink RJ, De Hingh IHJT, et al. PSOGI. Appendiceal tumours and pseudomyxoma peritonei: Literature review with PSOGI/EURACAN clinical practice guidelines for diagnosis and treatment. Eur J Surg Oncol 2020;50748-7983(20)30114-1.

5. Husain AN, Colby TV, Ordonez NG, et al. Guidelines for pathologic diagnosis of malignant mesothelioma: 2017 update of the consensus statement from the international mesothelioma interest group. Arch Pathol Lab Med 2017.

6. Carr NJ, Cecil TD, Mohamed F, et al. A consensus for classification and pathologic reporting of pseudo-myxoma peritonei and associated appendiceal neoplasia: the results of the peritoneal surface Oncology group international (PSOGI) modified Delphi process. Am J Surg Pathol 2016;40(1):14e26.

7. Hentzen JEKR, Constansia RDN, Been LB, et al. Diagnostic Laparoscopy as a Selection Tool for Patients with Colorectal Peritoneal Metastases to Prevent a Non-therapeutic Laparotomy During Cytoreductive Surgery. Ann Surg Oncol 2020;27(4):1084-93.

8. Sugarbaker PH. Peritonectomy procedures. Ann Surg 1995;221:29-42.

9. Jaquet $\mathrm{P}$, Sugarbaker $\mathrm{PH}$. Current methodologies for clinical assessment of patients with peritoneal carcinomatosis. J Exp Clin Cancer Res 1996;15:49-58.

10. González-Moreno S, Kusamura S, Baratti D, Deraco M. Postoperative residual disease evaluation in the locoregional treatment of peritoneal surface malignancy. J Surg Oncol 2008;98(4):237-41. PMID:18726884.

11. Kusamura $S$, Dominique $E$, Baratti $D$, et al. Drugs, carrier solutions and temperature in hyperthermic intraperitoneal chemotherapy. J Surg Oncol 2008;98(4):247-52.

12. Glehen O, Cotte E, Kusamura S, et al. Hyperthermic intraperitoneal chemotherapy: nomenclature and modalities of perfusion. J Surg Oncol 2008;98(4):242-6.

13. Pentheroudakis G, Pavlidis N. Serous papillary peritoneal carcinoma: Unknown primary tumour, ovarian cancer counterpart or a distinct entity? A systematic review. Critical Reviews in Oncol/Hematol 2010;75:27-42.

14. Chao KC, Chen YJ, Juang CM, et al. Prognosis for advanced-stage primary peritoneal serous papillary carcinoma and serous ovarian cancer in Taiwan. Taiwanese J Obs Gynecol 2013;52:81e84.

15. Choi CH, Kim TJ, Kim WY, et al. Papillary serous carcinoma in ovaries of normal size: A clinicopathologic study of 20 cases and comparison with extraovarian peritoneal papillary serous carcinoma. Gynecol Oncol 2007;105:762-8.

16. Yuan J, He L, Han B, Li Y. Long-term survival of high-grade primary peritoneal papillary serous. adenocarcinoma: a case report and literature review. World J Surg Oncol 2017;15(1):76. Doi: 10.1186/s12957-017-1134-3.

17. Bakrin N, Gilly FN, Baratti D, et al. Primary peritoneal serous carcinoma treated by cytoreductive surgery combined with hyperthermic intraperitoneal chemotherapy. A multi-institutional study of 36 patients. Eur J Surg Oncol 2013;39:742-7.

18. Sinukumar $S$, Kusamura $S$, Baratti $D$, et al. Improved survival with cytoreductive surgery, total parietal peritonectomy and hyperthermic intraperitoneal chemotherapy for serous papillary peritoneal carcinoma largest single institute experience. Poster Presentation at the $11^{\text {th }}$ International Workshop on Peritoneal Surface Malignancy Paris September 9-11, 2018.

19. Deraco M, Sinukumar S, Salcedo-Hernandez RA, et al. Clinic-pathological outcomes after total parietal peritonectomy, cytoreductive, Gil Gomez E. Cytoreductive surgery and hyperthermic intraperitoneal chemotherapy in advanced for serous papillary peritoneal papillary carcinoma submitted to neoadjuvant systemic chemotherapy- largest single institute experience. Eur J Surg Oncol 2019;45(11):2103-8.

20. McBride K, McFadden D, Osler T. Improved survival of patients with pseudomyxoma peritonei 
receiving intraperitoneal chemotherapy with cytoreductive surgery: a systematic review and meta-analysis. J Surg Res 2013;183(1):246-52.

21. Rossi CR, Foletto M, Mocellin S, et al. Hyperthermic intraoperative intraperitoneal chemotherapy with cisplatin and doxorubicin in patients who undergo cytoreductive surgery for peritoneal carcinomatosis and sarcomatosis: phase I study. Cancer 2002;94(2):492-9.

22. Bijelic L, Stuart OA, Sugarbaker PH. Adjuvant bidirectional chemotherapy with intraperitoneal pemetrexed combined with intravenous cisplatin for diffuse malignant peritoneal mesothelioma. Gastroenterol Res Pract Volume 2012;ID 890450.

23. Alexander HR Jr, Bartlett DL, Pingpank JF, et al. Treatment factors associated with long-term survival after cytoreductive surgery and regional chemotherapy for patients with malignant peritoneal mesothelioma. Surgery 2013;153:779-86.

24. Malgras B, Gayat E, Aoun O, et al. Impact of Combination Chemotherapy in Peritoneal Mesothelioma Hyperthermic Intraperitoneal Chemotherapy (HIPEC): The RENAPE Study. Ann Surg Oncol 2018. Doi: 10.1245/s10434-018-6631-2.

25. Helm JH, Miura JT, Gleen JA, et al. Cytoreductive surgery and hyperthermic intraperitoneal chemotherapy for malignant peritoneal mesothelioma: a systematic review and meta-analysis. Ann Surg Oncol 2015;22:1686-93.
26. Deraco M, Baratti D, Hutanu I, et al. The role of perioperative systemic chemotherapy in diffuse malignant peritoneal mesothelioma patients treated with cytoreductive surgery and hyperthermic intraperitoneal chemotherapy. Ann Surg Oncol 2013;20:1093-100.

27. Robella M, Vaira M, Mellano A, et al. Treatment of diffuse malignant peritoneal mesothelioma (DMPM) by cytoreductive surgery and HIPEC. Minerva Chirurgica 2014;69(1):9-15.

28. Piso P, Glockzin G, Von Breitenbuch, et al. Quality of Life After Cytoreductive Surgery and Hyperthermic Intraperitoneal Chemotherapy for Peritoneal Surface Malignancies. J Surg Oncol 2009;100:317-20.

29. Van Driel WJ, Koole SN, Sonke GS. Hyperthermic Intraperitoneal Chemotherapy in Ovarian Cancer. N Engl J Med 2018;378(14):1363-4.

30. Cashin PH, Mahteme $\mathrm{H}$, Spang $\mathrm{N}$, et al. Cytoreductive surgery and intraperitoneal chemotherapy versus systemic chemotherapy for colorectal peritoneal metastases: A randomised trial. Eur J Cancer 2016;53:155-62.

31. Rudloff $U$, Langan RC, Mullinax JE, et al. Impact of maximal cytoreductive surgery plus regional heated intraperitoneal chemotherapy (HIPEC) on outcome of patients with peritoneal carcinomatosis of gastric origin: results of the GYMSSA trial. J Surg Oncol 2014;110(3):275-84. 